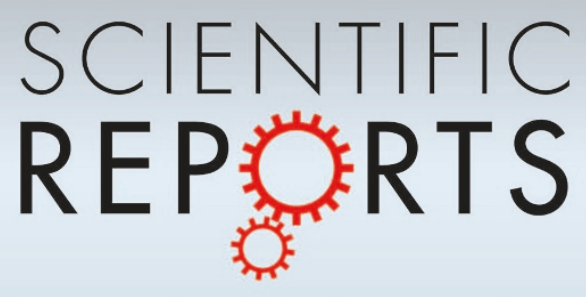

OPEN

SUBJECT AREAS:

WIDE-FIELD

FLUORESCENCE

MICROSCOPY

FLUORESCENCE SPECTROMETRY

Received

23 July 2014

Accepted

31 December 2014

Published

2 February 2015

Correspondence and requests for materials should be addressed to

A.H.A.C. (aclayton@ swin.edu.au)

\title{
The transition from single molecule to ensemble revealed by fluorescence polarization.
}

\author{
Toby D. M. Bell' \& Andrew H. A. Clayton ${ }^{2}$
}

\begin{abstract}
${ }^{1}$ School of Chemistry, Faculty of Science, Monash University, Clayton, Victoria 3800, Australia, ${ }^{2}$ Centre for Micro-Photonics, School of Science, Faculty of Science, Engineering and Technology, Swinburne University of Technology, Hawthorn, Victoria 3122, Australia.
\end{abstract}

Fluorescence polarization measurements in the condensed phase provide rich information on rotational dynamics and interactions between macromolecules. An important parameter in these studies is the limiting polarization or $p_{o}$ which is the emission polarization in the absence of molecular rotation. Here we explore how molecular number averaging affects the observed value of $p_{0}$. Using a simple mathematical model we show that for a collection of fluorescent dipoles (1-50 molecules) the fluorescence polarization ( $p$ ) increases with the number of molecules $(\mathrm{N})$ due to the progressive onset of photo-selection with a relation of the form $p=p_{o}\left(1-N^{-\beta}\right)$. This concept is demonstrated experimentally using single molecule polarization measurements of perylene diimide dye molecules in a rigid polymer matrix where it is shown that the average emission polarization increases significantly when the number of molecules per averaging window is increased from 1 to 10 molecules. These results suggest that the definition of limiting polarization needs to be refined in the quasi-single molecule regime. Moreover, these results pave a new way for measuring clustering of molecules from single cluster polarization histograms.

- mission polarization, also related to emission anisotropy, is a quantity that defines the orientation distribution of emitting molecules during their excited-state lifetime ${ }^{1}$. It is widely used in the physical and life sciences in rotational diffusion measurements as a probe of the size, shape, and flexibility of macromolecules ${ }^{2,3}$.

For a spherical rotator with rotational relaxation time $\rho$, the steady-state emission polarization $p$ is given by the Perrin equation ${ }^{1,2}$,

$$
((1 / \mathrm{p})-(1 / 3))=\left(\left(1 / \mathrm{p}_{\mathrm{o}}\right)-(1 / 3)\right)(1+3 \tau / \rho)
$$

Where $\tau$ is the fluorophore excited-state lifetime and $\mathrm{p}_{\mathrm{o}}$ is the emission polarization in the absence of molecular rotation. Changes in size (dimerization) or shape (conformation) can be observed through changes to the rotational relaxation time, $\rho$ and correspondingly, the polarization (or anisotropy). For a dilute solution of molecules in the micro-molar range the limiting polarization is generally considered to be a constant, only dependent on the angle between the absorption dipole and the emission dipole.

With single molecule techniques it is now possible to image individual molecules and clusters of molecules ${ }^{4-9}$. In this paper, we consider how the limiting polarization $\mathrm{p}_{\mathrm{o}}$ is influenced by the number of molecules, in other words in the quasi-single molecule or "few molecule" limit. We will deal with the case where molecules are rotationally-immobile and randomly-oriented, in the limit of $\tau / \rho \rightarrow 0$ in equation (1), but make the additional assumptions that (i) the molecules are independent emitters and absorbers (ii) do not undergo homo-transfer and (iii) do not rotate during the measurement time.

The concept of a number-dependent limiting polarization can be introduced by considering the number of molecules confined to a unit area or pixel. If the spatial resolution is sufficient to detect single molecules, then averaging a large number of single molecule polarizations will produce a net result of zero (Figure 1A). If each defined pixel or area contains 2 or more emitting molecules, then photo-selection will ensue and a net polarization will be observed (Figure 1B). For large numbers of emitting molecules per minimum pixel or area, the net polarization attains the bulk value of 0.5 (Figure 1C).

To address this quantitatively, we set up a simple model comprising fixed dipoles lying flat on a surface and computed the emission polarization distributions for different numbers of dipoles, from 1 to 50 dipoles. We show 
A
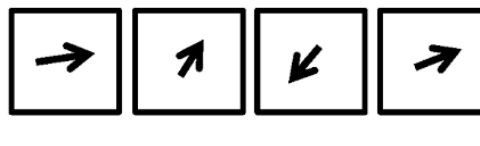

$P=0$

B
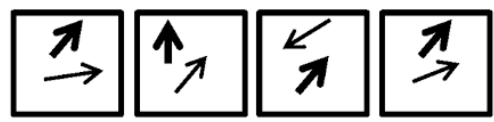

$\mathrm{P}>0$

C
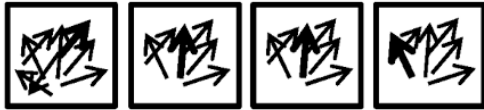

$P=0.5$

Figure 1 1 Schematic concept of number-dependent polarization (a) Sample of single molecules oriented randomly on a plane. Polarizations can be both positive and negative (no photoselection) then the average polarization sums to zero. (b) Samples of two molecules per unit area. The molecule oriented towards the excitation polarization is preferentially excited (photoselected). As a consequence the average polarization is finite. (c) Many molecules per area. Here there are always molecules oriented nearly parallel to the excitation. Then the polarization approaches the maximum value.

that for single dipoles the average emission polarization is close to zero, as expected ${ }^{9}$ and that at 50 dipoles per averaging-window the emission polarization is 0.497 , very close to the limiting emission polarization of 0.5 for the bulk case. Both the calculated mean and variance of the emission polarization distribution are very sensitive in the range of 2-10 molecules where a sharp increase in polarization with increasing molecule number is predicted.

To provide experimental support for our findings we report single molecule polarization imaging measurements ${ }^{11}$ of a fluorescent dye in a thin $(\sim 200 \mathrm{~nm})$ and rigid, transparent polymer film. As expected, the emission polarization of single molecules averages to nearly zero, in contrast to the bulk emission polarization of $\sim 0.5$ from a concentrated solution. Using progressive photobleaching to vary the number of emitting molecules per region of imaging we show that bleaching from $<100$ dipoles to a few dipoles results in a dramatic decrease in the average emission polarization, consistent with the theoretical predictions. These results suggest that the textbook view of fundamental polarization needs to be refined. Moreover the calculations and experiments suggest a new means to determine cluster sizes (or numbers) of molecules from emission polarization images or histograms.

\section{Results}

When a fluorescent sample is illuminated with plane-polarized light, the emission polarization ( $p$ ) is defined by the relative emission detected through a polarizer aligned parallel to the excitation polarization direction $\left(\mathrm{I}_{\text {par }}\right)$ and the emission detected through a polarizer aligned perpendicular $\left(\mathrm{I}_{\text {perp }}\right)$ to the excitation polarization direction.

$$
\mathrm{p}=\left(\mathrm{I}_{\text {par }}-\mathrm{I}_{\text {perp }}\right) /\left(\mathrm{I}_{\text {par }}+\mathrm{I}_{\text {perp }}\right)
$$

The emission anisotropy ( $r$ ) is also a useful parameter to characterize the extent of the polarization of the emission. In what follows we present our results using polarization values but the reader can readily convert polarization to anisotropy with equation (3).

$$
\mathrm{r}=\left(\mathrm{I}_{\mathrm{par}}-\mathrm{I}_{\text {perp }}\right) /\left(\mathrm{I}_{\mathrm{par}}+2 \mathrm{I}_{\text {perp }}\right)=2 \mathrm{p} /(3-\mathrm{p})
$$

Consider a single dipole lying flat on a plane, fixed in orientation at an angle $\phi$ with respect to the excitation polarization (Figure 2A).

The probability for (single) photon absorption is proportional to $\cos ^{2} \phi$

$$
\operatorname{Prob}(\phi)=\mathrm{k}_{1} \cos ^{2} \phi
$$

In equation $3, \mathrm{k}_{1}$ is a constant of proportionality dependent on the experimental set-up and molecule of interest. For co-linear absorption and emission dipoles, the polarized components of the emission $\left(\mathrm{I}_{\text {par }}, \mathrm{I}_{\text {perp }}\right)$ are given by,

$$
\begin{gathered}
\mathrm{I}_{\mathrm{par}}=\mathrm{k}_{2} \operatorname{Prob}(\phi) \cos ^{2} \phi=\mathrm{k}_{1} \mathrm{k}_{2} \cos ^{4} \phi \\
\mathrm{I}_{\text {perp }}=\mathrm{k}_{2} \operatorname{Prob}(\phi) \sin ^{2} \phi=\mathrm{k}_{1} \mathrm{k}_{2} \cos ^{2} \phi \sin ^{2} \phi
\end{gathered}
$$

In equations 5 and $6 \mathrm{k}_{2}$ is a constant of proportionality dependent on the experimental configuration. Substituting equations (5) and (6) into equation (2) yields the expression for the polarization of a single dipole as a function of angle $\phi$,

$$
\begin{aligned}
\mathrm{p} & =\left(\mathrm{I}_{\text {par }}-\mathrm{I}_{\text {perp }}\right) /\left(\mathrm{I}_{\text {par }}+\mathrm{I}_{\text {perp }}\right) \\
& =\left(\cos ^{2} \phi-\sin ^{2} \phi\right) /\left(\cos ^{2} \phi+\sin ^{2} \phi\right)=\cos (2 \phi)
\end{aligned}
$$

By inspection of equation (7), it is clear that a single dipole flat on a surface can assume any emission polarization in the range -1 to 1 , as
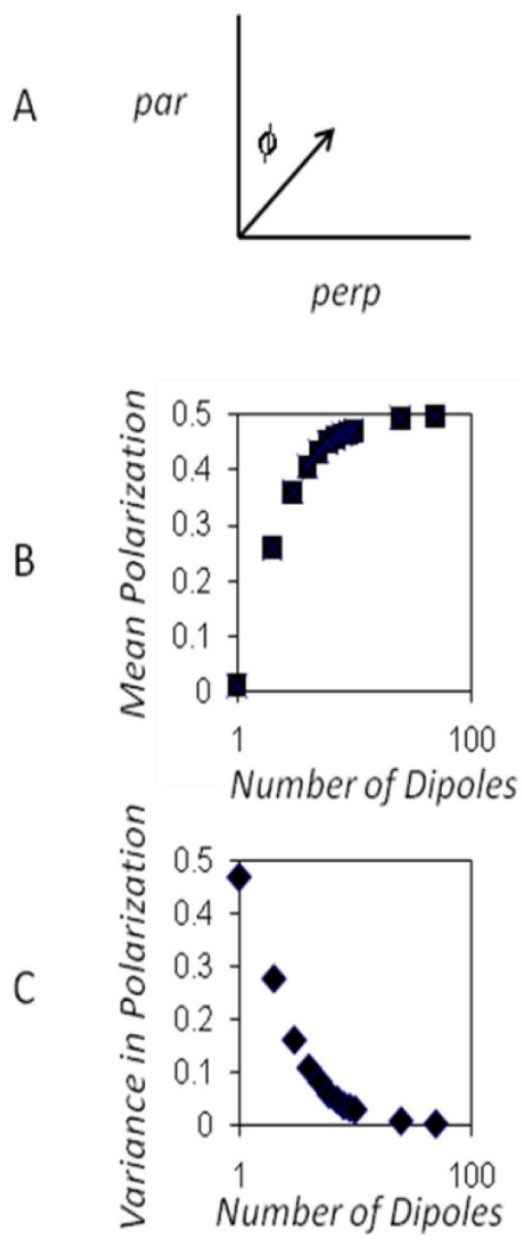

Figure $2 \mid$ (a) Geometry for idealized dipole lying on a flat plane. The direction of excitation polarization and direction of emission polarizations parallel and perpendicular to the excitation polarization are indicated. Optical axis is normal to the plane of the page. (b) Theoretical mean polarization as a function of dipole number from simulation of 1000 runs (see text for details). (c) Theoretical variance in the polarization distribution from simulation of 1000 runs (see text for details). 
expected, since $\phi$ can be assigned any value from 0 to $2 \pi$. Since the cosine function is symmetric i.e. the probability of having a dipole with negative polarization is equally likely to a dipole with positive polarization it is obvious that the average polarization for the physical model described (no photo-selection) will be zero. This can be confirmed by integrating equation (7) with respect to $\phi$ over the limits of $\pi$ to zero.

For two dipoles, dipole $\mathrm{A}$ and dipole $\mathrm{B}$, equations (5) and (6) become equations (8) and (9),

$$
\begin{aligned}
& \mathrm{I}_{\mathrm{par}}=\mathrm{k}_{2}\left(\left(\operatorname{Prob}\left(\phi_{\mathrm{A}}\right) \cos ^{2} \phi_{\mathrm{A}}\right)+\left(\operatorname{Prob}\left(\phi_{\mathrm{B}}\right) \cos ^{2} \phi_{\mathrm{B}}\right)\right) \\
& \mathrm{I}_{\text {perp }}=\mathrm{k}_{2}\left(\left(\operatorname{Prob}\left(\phi_{\mathrm{A}}\right) \sin ^{2} \phi_{\mathrm{A}}\right)+\left(\operatorname{Prob}\left(\phi_{\mathrm{B}}\right) \sin ^{2} \phi_{\mathrm{B}}\right)\right.
\end{aligned}
$$

Where, in equations 8 and 9, we now have two angles, $\phi_{\mathrm{A}}$ for dipole $\mathrm{A}$ and $\phi_{\mathrm{B}}$ for dipole $\mathrm{B}$.

The emission polarization of a double (independent) dipole system is then given by

$$
\begin{aligned}
\mathrm{p}\left(\phi_{\mathrm{A}}, \phi_{\mathrm{B}}\right)= & {\left[\cos ^{2}\left(\phi_{\mathrm{A}}\right) \cos \left(2 \phi_{\mathrm{A}}\right)+\cos ^{2}\left(\phi_{\mathrm{B}}\right) \cos \left(2 \phi_{\mathrm{B}}\right)\right] / } \\
& {\left[\cos ^{2}\left(\phi_{\mathrm{A}}\right)+\cos ^{2}\left(\phi_{\mathrm{B}}\right)\right] }
\end{aligned}
$$

By extrapolation, the emission polarization for $\mathrm{n}$-dipoles is

$$
\left.\left.\mathrm{p}\left(\phi_{\mathrm{A}}, \phi_{\mathrm{B}}, \ldots \phi_{\mathrm{N}}\right)=\left[\sum \cos ^{2}\left(\phi_{\mathrm{i}}\right) \cos \left(2 \phi_{\mathrm{i}}\right)\right] /\right] \sum \cos ^{2}\left(\phi_{\mathrm{i}}\right)\right]
$$

It is noted that in equation 11 the polarization not only depends on angle but also on the number of dipoles. By inspection it is clear that when there is one dipole, the $\cos ^{2}\left(\phi_{i}\right)$ term cancels and the polarization depends only on the angle. When there is more than one dipole, the dipole(s) aligned towards the parallel direction (i.e. larger $\left.\cos ^{2}\left(\phi_{\mathrm{i}}\right)\right)$ will dominate the summation biasing the polarization toward positive values. This can be illustrated using the numerical simulation results presented below.

Figure 2 summarizes the mean polarization (Figure 2B) and variance in the polarization (Figure 2C) from 1000 simulations of random dipoles ranging in number from 1 to 100 (applying equation (10) using randomly generated angles in Excel). Noteworthy is the transition from a mean polarization near zero for a single dipole to a value of 0.5 for 50 dipoles. This transition occurs sharply over the range 1-6 dipoles where the corresponding polarization increases from 0.01 to 0.26 ( 1 to 2 dipoles), to 0.36 ( 3 dipoles), to 0.41 (4 dipoles), and then from 0.43 to 0.45 ( 5 to 6 dipoles). The variance of the computed distribution is also quite informative. As expected, the variance is the largest (near 0.5) for single dipoles because there is no photo-selection, however the onset of photo-selection is apparent already for two dipoles with the variance reduced to 0.28 . At 50 dipoles the variance in the polarization distribution is $<0.005$.

To explore this molecule number effect experimentally we carried out single molecule polarization imaging experiments using a bright fluorophore (a perylene diimide derivative, PDI; structure in Figure 3B) spin-coated in a rigid poly(methylmethacrylate) (PMMA) polymer matrix. Figure $3 \mathrm{~A}$ reveals typical images from single molecules (at low dye loading such that single PDI molecules are well separated and their emission point-spread-functions (PSFs) are well resolved). From 70 individual spots or PSFs an average polarization was computed to be -0.02 , close to the expected value of zero. Importantly, individual polarization values were spread over a wide-range of values with lowest and highest values approaching the expected extremes of -1 and 1 in line with previously reported distributions for single molecule fluorescence ${ }^{9}$.

To investigate the effect of number of dipoles on the magnitude of the emission polarization, we depleted dipoles photochemically and measured the number of molecules emitting using image correlation
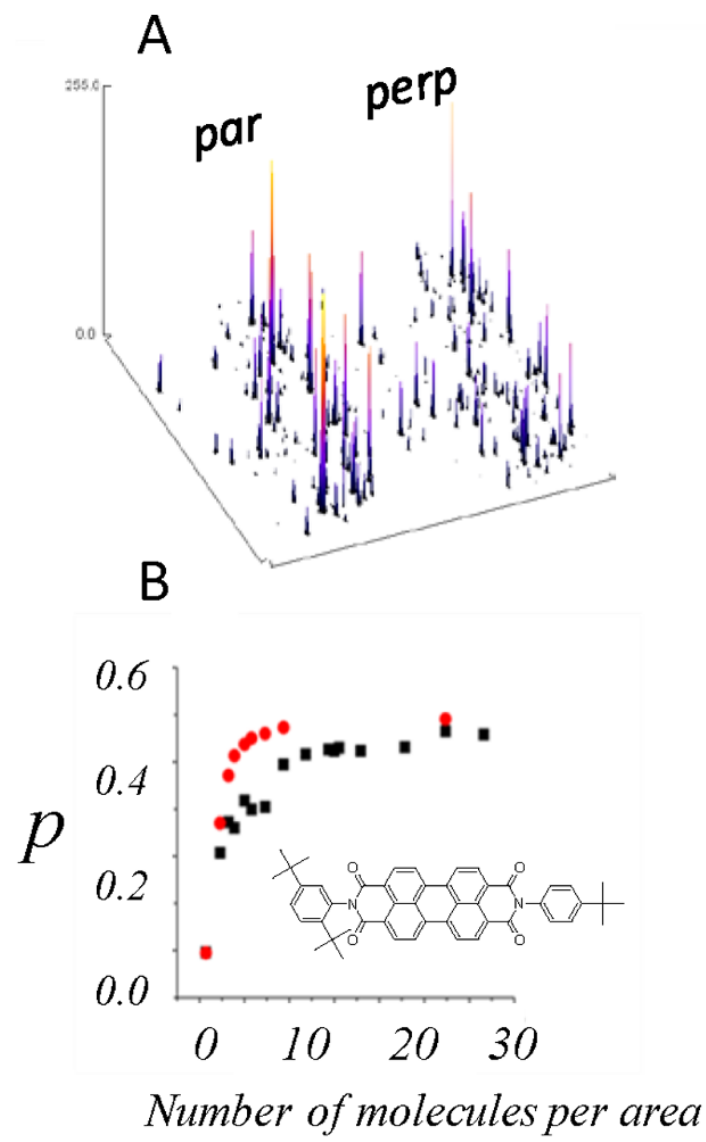

Figure $3 \mid$ (a) Images of the parallel and perpendicular polarized components of the emission from the single PDI dye molecules in a PMMA matrix. (b) Mean fluorescence polarization as a function of number of fluorophores for a collection of single dye molecules in a polymer matrix. Note the decrease in average emission polarization with reduction in number of molecules imaged (data, black squares). Red circles represent simulation results. Inset: chemical structure of the PDI dye.

spectroscopy ${ }^{10}$. Figure $2 \mathrm{~A}$ is a typical distribution before photobleaching. A typical plot of polarization as a function of observed molecules is shown in Figure 3B (black symbols). It is clear that with 2 molecules the polarization has increased by 0.22 (from -0.02 to 0.20 ) very close to the predicted increase of 0.25 from simulations. However, as the number of detected molecules increases the polarization also increases more gradually, as predicted from theory (theory (red dots), experiment (black dots), Figure 3B). At 10 molecules and greater, the polarization begins to plateau at around 0.4 with some small fluctuations in value from 10 to 30 molecules. The deviations between experiment and theory may be due to a small proportion of molecules tilted out of the plane, photo-selected photobleaching and additional depolarizing effects due to the high NA objective ${ }^{11}$. For the $\sim 200 \mathrm{~nm}$ thin-film created here, we expect greater than $80 \%$ of molecules oriented in the plane of the glass coverslip. Depolarization can reduce polarizations by as much as $20 \%{ }^{11}$.

At high dye loading, the value of the emission polarization should be well approximated by the limiting polarization value of 0.5 . From the corrected average intensities of the parallel and perpendicularpolarized components of the emission from a 'quasi-bulk' film (containing sufficient PDI molecules to ensure complete coverage of emission), the polarization was estimated to be $0.48 \pm 0.01$ (data not shown).

To our knowledge, this is the first demonstration that the average emission polarization for a rotationally-immobile set of molecules is 
positively correlated with the number of molecules in the observation area. The transition from the near zero polarization observed for single molecules to the limiting polarization at 0.5 at 50 or more molecules occurs over a range from 2-10 molecules. In is important to note that this effect is restricted to fully rigid molecules which are randomly oriented and which do not undergo homo-transfer. Lack of molecular rigidity will also reduce the effect discussed here since rotation on the fluorescence time-scale will reduce the polarization. Rotation on the measurement time-scale will produce a photo-selection effect, even for single molecules that are immobile on the fluorescent timescale ${ }^{8}$. One caveat is that molecules which are highly ordered in orientation with respect to excitation polarization will have equal (or very similar) probability of photo-selection and are unlikely to display the phenomenon considered in this paper. Homotransfer which involves exchange of non-radiative electronic energy between molecules breaks the assumption of independent absorbers and emitters.

Molecules on biological cell surfaces have typical densities of between 0.1 to 100 molecules per square micron ${ }^{10}$. Supramolecular complexes important for biological function typically contain 1-10 molecules ${ }^{10}$. Quasi-single molecule polarization on appropriately rigidified fluorophores has the potential to determine molecular densities and cluster sizes of cell surface molecules. Provided that (i) the emission polarization of individual particles (or clusters) can be resolved and (ii) that fluorophores within the clusters are noninteracting ( $>10 \mathrm{~nm}$ apart) and immobile, the particle polarization histogram can provide information on the mean number of dipoles per particle (or cluster) or mean number of dipoles within a larger imaging area. For $\mathrm{N}$ fluorophores per area, the average polarization (from theory) from a large number of areas will be approximately $\mathrm{p}$ $=0.5\left(1-\left(\mathrm{N}^{-1.2}\right)\right)$.

\section{Experimental Protocol}

Materials and Methods. Fluorescence images were recorded using an inverted epifluorescence microscope (Olympus IX71) equipped with a high NA objective (Olympus Planfluo 1.3 NA 100X) and imaged on an EMCCD camera (Princeton ProEm512) after passing through a polarizing beam-splitting device (Dual-viewer, Optical Insights, Tuscon, USA). Excitation at $532 \mathrm{~nm}$ was provided by a $300 \mathrm{~mW} \mathrm{cw}$ solid state diode laser (Dragon Lasers Inc), expanded using telescopic lenses (Thor Labs Pty Ltd) and focused to the back of the objective (Köhler illumination) using a $500 \mathrm{~mm}$ lens (Thor Labs Pty. Ltd.) so that the area of illumination was approximately $30 \mu \mathrm{m}$ in diameter. Scattered excitation light was excluded from the detected light by dichroic (z532rdc) and long pass (HQ545LP) filters (Chroma).

Bulk and single molecule samples were prepared on cleaned glass coverslips by spincoating (SC600, Laurell Technologies, USA) solutions of the perylene diimide dye in $\sim 5 \mathrm{mg} / \mathrm{ml} \mathrm{PMMA}$ (Aldrich, $\mathrm{Mw} 350000, \mathrm{Tg} 122^{\circ} \mathrm{C}$ ) in spectroscopic grade $\mathrm{CHCl}_{3}$ (Aldrich). Bulk samples were prepared from $\sim \mu \mathrm{M}$ dye concentration solutions whereas for single molecule measurements, solutions were diluted to $\sim \mathrm{nM}$.
1. Weber, G. Polarization of the fluorescence of macromolecules. I. Theory and experimental method. Biochem J 51, 145-155 (1952).

2. Weber, G. Polarization of the fluorescence of macromolecules. II. Fluorescent conjugates of ovalbumin and bovine serum albumin. Biochem J 51, 155-167 (1952).

3. Jameson, D. M. \& Ross, J. A. Fluorescence polarization/anisotropy in diagnostics and imaging. Chem Rev 110, 2685-2708 (2010).

4. Schultz, G. J., Schindler, H. \& Schmidt, Th. Single molecule dichroism. Opt. Lett. 22, 561-564 (1997).

5. Dickson, R. M., Norris, D. J. \& Moerner, W. E. Simultaneous imaging of individual molecules aligned both parallel and perpendicular to the optical axis. Phys. Rev. Lett. 24, 5322-5325 (1998).

6. Ha, T., Glass, J., Enderle, Th., Chemla, D. S. \& Weiss, S. Hindered rotational diffusion and jumps of single molecules. Phys. Rev. Lett. 80, 2093-2096 (1998).

7. Rosenberg, S. A., Quinlan, M. E., Forkey, J. N. \& Goldman, Y. E. Rotational motions of macro-molecules by single-molecule fluorescence microscopy. Acc Chem Res 38, 583-593 (2005).

8. Harms, G. S., Sonnleitner, M., Schutz, G. J., Gruber, H. J. \& Schmidt, T. Singlemolecule anisotropy imaging. Biophys J 77, 2864-2870 (1999).

9. Chung, I., Shimizu, K. T. \& Bawendi, M. G. Room temperature measurements of the $3 \mathrm{D}$ orientation of single CdSe quantum dots using polarization microscopy. PNAS 100, 405-408 (2003).

10. Wiseman, P. W. \& Petersen, N. O. Image correlation spectroscopy. II. Optimization for ultrasensitive detection of preexisting platelet-derived growth factor-beta receptor oligomers on intact cells. Biophys J., 76, 963-977 (1998).

11. Axelrod, D. Fluorescence polarization microscopy. Methods Cell Biol 30, 333-352 (1989).

\section{Acknowledgments}

TDMB acknowledges the ARC for funding through grants LE100100131 and LE110100161, and Monash University Faculty of Science for a Dean's strategic funding initiative. AHAC acknowledges ARC funding through DP110100164 and DP130101475 grants. We thank Dr. Ming Chen for the generous gift of the perylene diimide compound.

\section{Author contributions}

T.D.M.B. and A.H.A.C. performed experiments and/or analysed data; T.D.M.B. and A.H.A.C. designed the research; T.D.M.B. and A.H.A.C. wrote the paper.

\section{Additional information}

Competing financial interests: The authors declare no competing financial interests.

How to cite this article: Bell, T.D.M. \& Clayton, A.H.A. The transition from single molecule to ensemble revealed by fluorescence polarization.. Sci. Rep. 5, 8158; DOI:10.1038/ srep08158 (2015)

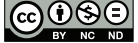

This work is licensed under a Creative Commons Attribution-NonCommercialNoDerivs 4.0 International License. The images or other third party material in this article are included in the article's Creative Commons license, unless indicated otherwise in the credit line; if the material is not included under the Creative Commons license, users will need to obtain permission from the license holder in order to reproduce the material. To view a copy of this license, visit http:// creativecommons.org/licenses/by-nc-nd/4.0/ 